\title{
AN INTEGRAL EQUATION APPROACH TO THE INCOMPRESSIBLE NAVIER-STOKES EQUATIONS IN TWO DIMENSIONS*
}

\author{
LESLIE GREENGARD ${ }^{\dagger}$ AND MARY CATHERINE KROPINSKI ${ }^{\ddagger}$
}

\begin{abstract}
We present a collection of methods for solving the incompressible Navier-Stokes equations in the plane that are based on a pure stream function formulation. The advantages of this approach are twofold: first, the velocity is automatically divergence free, and second, complicated (nonlocal) boundary conditions for the vorticity are avoided. The disadvantage is that the solution of a nonlinear fourth-order partial differential equation is required. By recasting this partial differential equation as an integral equation, we avoid the ill-conditioning which hampers finite difference and finite element methods in this environment. By using fast algorithms for the evaluation of volume integrals, we are able to solve the equations using $O(M)$ or $O(M \log M)$ operations, where $M$ is the number of points in the discretization of the domain.
\end{abstract}

Key words. Navier-Stokes equations, integral equation methods

AMS subject classifications. 31A10, 65R20, 76D05

PII. S1064827597317648

1. Introduction. At the present time, methods for solving large-scale problems in incompressible fluid dynamics are based largely on finite difference or finite element methods. While enormous advances have been made in these approaches over the past few decades, a number of open questions remain. These include the discretization of complex flow domains, the imposition of the incompressibility constraint, the selection of boundary conditions, and the resolution of complex solution features away from the boundary. An equally important issue is the development of good turbulence models, but we concern ourselves in this paper with flows that can be fully resolved and assume the Reynolds numbers Re ranges up to the order of one thousand. In some sense, this paper is an extension of our previous work on fast algorithms for the Stokes equations in complex geometries [10], for which the Reynolds number is zero.

Integral equation methods offer several advantages in this setting, namely, that complex physical boundaries are easy to incorporate and that the ill-conditioning associated with direct discretization of the governing partial differential equations is avoided. Nevertheless, despite some previous work [24, 29], such an approach has been deemed impractical as a general tool because of the apparent computational expense. The reason is clear. For any problem other than the Stokes equations, unknowns are distributed at all points of the domain. On an $N \times N$ mesh, the integral formulation leads to dense $N^{2} \times N^{2}$ matrices.

We hope to demonstrate here that, in fact, integral equation methods provide a sound basis for the investigation of both steady and unsteady flows over a fairly wide range of Reynolds numbers. We express the Navier-Stokes equations as a nonlinear

\footnotetext{
*Received by the editors March 3, 1997; accepted for publication August 6, 1997; published electronically August 5, 1998.

http://www.siam.org/journals/sisc/20-1/31764.html

${ }^{\dagger}$ Courant Institute of Mathematical Sciences, New York University, New York, NY 10012 (greengard@cims.nyu.edu). The work of this author was supported by the Applied Mathematical Sciences Program of the U.S. Department of Energy under contract DEFGO288ER25053, by a NSF Presidential Young Investigator Award, and by a Packard Foundation Fellowship.

${ }^{\ddagger}$ Department of Mathematics and Statistics, Simon Fraser University, Burnaby, BC, Canada V5A 1S6 (mkropins@cs.sfu.ca). The work of this author was supported by NSERC grant WFA 0172672.
} 
fourth-order partial differential equation satisfied by the stream function $\psi$, and solve this equation in an iterative fashion. For steady flows, the integral representation for $\psi$ involves layer and volume potentials based on fundamental solutions for the biharmonic equation (Stokes flow). For unsteady flows, a modified biharmonic Green's function is used. In order to focus our attention on the behavior of the integral equation approach and the performance of various iteration schemes, we restrict our attention to flow inside a circular cylinder. Complex domains will be considered at a later date [11].

For highly viscous steady flows $(R e<5)$, a simple fixed-point iteration scheme is very effective and requires only the solution of a sequence of forced biharmonic boundary value problems. For higher Reynolds numbers $(5<R e<3000)$, we have chosen to use an inexact Newton method [7], which converges rapidly but requires the solution of a more complicated, nonconstant coefficient, fourth-order partial differential equation at each iteration. For unsteady flows, the equations of motion are evolved in time using linearly implicit or implicit-explicit (IMEX) methods, which require the solution of a modified biharmonic equation at each time step.

While flow past a circular cylinder represents a classical problem in both theoretical and computational fluid dynamics, relatively little attention has been given to flow inside a cylinder. Most published studies appear to be based on the stream function/vorticity formulation of the two-dimensional Navier-Stokes equations, which requires boundary conditions for the vorticity. At low to moderate Reynolds numbers, this results in either nonlocal boundary conditions or schemes with low-order accuracy and poor stability properties [1]. Interesting previous studies include those of Kuwahara and Imai [16], who use a finite difference method, Panikker and Lavan [25], who use a hybrid finite difference/integral equation method, Dennis, Ng, and Nguyen [6], who use a Fourier spectral method, Wang and Wu [30], who use an integrodifferential equation, and Huang and Tang [14], who use a hybrid finite difference/pseudospectral method. The approach most closely related to ours is that of Mills [22], who developed an integral equation method based on the biharmonic Green's function for a cylinder. This approach, however, does not extend to more complex flow domains, where the exact Green's function is unavailable. In the approach described below, the stream function is expressed in terms of volume and boundary source distributions using an easily computed Green's function, so that no geometric restrictions on the flow domain apply.

The paper is organized as follows: section 2 reviews the equations of motion for incompressible, viscous flow and section 3 contains a discussion of the basic numerical tools we will require. In sections 4 and 5 , we incorporate these tools into fixed-point and Newton iterations for the steady Navier-Stokes equations, and in section 6, we describe methods for the unsteady problem. Numerical examples are presented in section 7 , and we end with a brief discussion.

2. The governing equations. We consider the incompressible Navier-Stokes equations in the standard nondimensional form

$$
\begin{aligned}
\frac{\partial \mathbf{u}}{\partial t}+(\mathbf{u} \cdot \nabla) \mathbf{u} & =-\nabla p+\frac{1}{R e} \Delta \mathbf{u}, \\
\nabla \cdot \mathbf{u} & =0
\end{aligned}
$$

where $\mathbf{u}$ is the velocity vector, $p$ is the pressure, and $R e$ is the Reynolds number of the flow. An additional quantity of interest is the vorticity $\boldsymbol{\omega}=\nabla \times \mathbf{u}$. Equation (1) represents a balance of viscous, inertial, and pressure forces, and (2) is the continuity 
equation; together they constitute a system of equations for the unknowns $\mathbf{u}$ and $p$. For two-dimensional flow in polar coordinates, this system is represented more compactly by introducing the scalar stream function $\Psi(r, \theta, t)$, where

$$
u_{r}=\frac{1}{r} \frac{\partial \Psi}{\partial \theta}, \quad u_{\theta}=-\frac{\partial \Psi}{\partial r}
$$

The continuity equation (2) is then satisfied automatically. Taking the curl of both sides of (1), the Navier-Stokes equations reduce to a single fourth-order equation

$$
\frac{\partial \Delta \Psi}{\partial t}+J_{r}[\Psi, \Delta \Psi]=-\frac{1}{R e} \Delta^{2} \Psi
$$

where $J_{r}$ is the Jacobian defined by $J_{r}[a, b]=\frac{1}{r}\left(\partial_{r} a \partial_{\theta} b-\partial_{\theta} a \partial_{r} b\right)$ and the Laplacian operator $\Delta$ in polar coordinates is

$$
\Delta \equiv \frac{\partial^{2}}{\partial r^{2}}+\frac{1}{r} \frac{\partial}{\partial r}+\frac{1}{r^{2}} \frac{\partial^{2}}{\partial \theta^{2}} .
$$

The vorticity vector points in the direction normal to the $(r, \theta)$ plane and can be represented by a scalar function

$$
\omega=-\Delta \Psi .
$$

We will restrict our attention in this paper to flow in the interior of a unit disk, with the velocity specified on the boundary $r=1$. Standard boundary conditions are then of the form

$$
u_{r}(1, \theta, t)=f^{2}(\theta, t), \quad u_{\theta}(1, \theta, t)=-f^{1}(\theta, t) .
$$

In terms of the stream function, these become

$$
\nabla \Psi(1, \theta, t)=\left(f^{1}(\theta, t), f^{2}(\theta, t)\right)
$$

where

$$
\nabla \equiv\left(\frac{\partial}{\partial r}, \frac{1}{r} \frac{\partial}{\partial \theta}\right)
$$

One cannot, however, assign an arbitrary velocity distribution on the boundary. In order that mass be conserved, there must be zero net flux across the boundary of the disk:

$$
\int \mathbf{u} \cdot \mathbf{n} d S=\int_{0}^{2 \pi} u_{r}(1, \theta, t) d \theta=0
$$

In summary, for unsteady flows, we wish to solve the following:

$$
\begin{array}{cc}
\frac{\partial \Delta \Psi}{\partial t}+J_{r}[\Psi, \Delta \Psi]=-\frac{1}{R e} \Delta^{2} \Psi & \text { for } r<1, \\
\nabla \Psi=\left(f^{1}(\theta, t), f^{2}(\theta, t)\right) & \text { for } r=1, \\
\Psi=\Psi_{0}(r, \theta) & \text { at } t=0 .
\end{array}
$$

For steady flows, the above reduces to

$$
\begin{array}{cc}
\Delta^{2} \Psi+\operatorname{Re} J_{r}[\Psi, \Delta \Psi]=0 & \text { for } r<1, \\
\nabla \Psi=\left(f^{1}(\theta), f^{2}(\theta)\right) & \text { for } r=1 .
\end{array}
$$


3. Numerical preliminaries. The methods we will develop for the full NavierStokes equations rely on solutions to the biharmonic and Poisson equations. In this section, we briefly describe some of the tools we will use to solve these boundary value problems in a disk.

3.1. Stokes flow. In creeping flow, $R e \rightarrow 0$ and the Navier-Stokes equations are approximated by the linear Stokes equations. In this limit, (5) reduces to the biharmonic equation:

$$
\begin{array}{cc}
\Delta^{2} \Psi=0 & \text { for } r<1, \\
\nabla \Psi=\left(f^{1}(\theta), f^{2}(\theta)\right) & \text { for } r=1 .
\end{array}
$$

Using the classical complex variable theory discussed by Mikhlin and others [17, $21,23,26]$, we note that any plane biharmonic function $\Psi(r, \theta)$ can be expressed by Goursat's formula as

$$
\Psi(r, \theta)=\operatorname{Re}(\bar{z} \phi(z)+\chi(z)),
$$

where $\phi$ and $\chi$ are analytic functions of the complex variable $z=r e^{i \theta}$, and $\operatorname{Re}(g)$ denotes the real part of the complex-valued function $g$. The functions $\phi(z)$ and $\psi(z)=$ $\chi^{\prime}(z)$ are known as Goursat functions. A simple calculation leads to Muskhelishvili's formula

$$
e^{i \theta} \frac{\partial \Psi}{\partial r}+i e^{i \theta} \frac{1}{r} \frac{\partial \Psi}{\partial \theta}=\phi(z)+z \overline{\phi^{\prime}(z)}+\overline{\psi(z)},
$$

providing an expression for the velocity field. The vorticity and pressure can also be expressed in terms of the Goursat functions by means of the relation

$$
-\omega+i p=4 \phi^{\prime}(z) .
$$

Muskhelishvili's formula (9) allows us to convert the problem of Stokes flow into a problem in analytic function theory, namely, that of finding $\phi$ and $\psi$ which satisfy appropriate conditions on the boundary. Letting $f(\theta)=f^{1}(\theta)+i f^{2}(\theta)$, we must simply ensure that

$$
\phi\left(e^{i \theta}\right)+e^{i \theta} \overline{\phi^{\prime}\left(e^{i \theta}\right)}+\overline{\psi\left(e^{i \theta}\right)}=e^{i \theta} f(\theta) .
$$

The natural compatibility condition (4), written in complex notation, is

$$
\operatorname{Im} \int_{0}^{2 \pi} f(\theta) d \theta=0
$$

where $\operatorname{Im} g$ denotes the imaginary part of the complex-valued function $g$.

While integral equation methods for obtaining the Goursat functions exist for general multiply connected domains [10], we describe a much simpler method for the unit disk. We first observe that since $\phi(z)$ and $\psi(z)$ are analytic, they can be represented by Taylor series:

$$
\begin{aligned}
\phi(z) & =\sum_{n=0}^{\infty} a_{n} z^{n}, & \chi(z) & =b_{0}+\sum_{n=2}^{\infty} \frac{b_{n-1}}{n} z^{n}, \\
\phi^{\prime}(z) & =\sum_{n=0}^{\infty}(n+1) a_{n+1} z^{n}, & \psi(z) & =\sum_{n=1}^{\infty} b_{n} z^{n} .
\end{aligned}
$$


If we assume that the complex velocity $f$ is tabulated at $2 N+1$ equispaced points on the boundary, it can be approximated with spectral accuracy in terms of a Fourier series

$$
f(\theta) \approx \sum_{n=-N}^{N} f_{n} e^{i n \theta}
$$

The coefficients $f_{n}$ in this truncated series can be obtained with the fast Fourier transform (FFT) using $O(N \log N)$ arithmetic operations. In order to determine the desired coefficients $a_{n}$ and $b_{n}$ in the Taylor series for $\phi$ and $\psi$, we substitute (12) into (10) and (11) and equate coefficients. This yields the system of equations

$$
\begin{array}{cc}
a_{n}=f_{n-1}, & 2<n \leq N, \\
a_{1}+\bar{a}_{1}=f_{0}, \quad a_{0}=f_{-1}-2 \bar{a}_{2}, & \\
b_{n}=\bar{f}_{-n+1}-(n+2) \bar{a}_{n+2}, & 1<n \leq N,
\end{array}
$$

which can be solved recursively using $O(N)$ operations. Once the Taylor coefficients have been determined, one can easily use the FFT to tabulate the solution at each point of a polar grid $\left(r_{i}, \theta_{j}\right)$, where $0<r_{1}<\cdots<r_{N_{r}}<1$ and $\theta_{j}=2 \pi(j-1) /(2 N+$ $1)$, at a total cost of $O\left(N_{r} N \log N\right)$ operations. With minor changes in notation, the method described here is equivalent to the series solution methods discussed in $[5,6,14,22,28,30]$.

3.2. A simple high-order Poisson solver. Consider now a Poisson equation of the form

$$
\begin{aligned}
\Delta u & =g(r, \theta) & & \text { for } r<1, \\
u & =0 & & \text { for } r=1 .
\end{aligned}
$$

Although standard solvers exist for this geometry, we briefly describe our approach, which is of arbitrary order accuracy and which allows for adaptivity in the radial direction. For this, we approximate $u(r, \theta)$ and $g(r, \theta)$ by the truncated Fourier expansions

$$
u(r, \theta) \approx \sum_{n=-N}^{N} u_{n}(r) e^{i n \theta}, \quad g(r, \theta) \approx \sum_{n=-N}^{N} g_{n}(r) e^{i n \theta},
$$

where $u_{n}(r)$ and $g_{n}(r)$ are complex-valued functions. Substituting these expressions into (14) leads to the following sequence of two-point boundary value problems:

$$
\left.\begin{array}{c}
u_{n}^{\prime \prime}+\frac{1}{r} u_{n}^{\prime}-\frac{n^{2}}{r^{2}} u_{n}=g_{n}(r) \quad \text { for } 0<r<1, \\
u_{0}^{\prime}(0)=0 \\
u_{n}(0)=0, n \neq 0
\end{array}\right\}, u_{n}(1)=0 .
$$

For each Fourier mode $n$, we solve the equation (15) using an adaptive, high-order solver described in $[12,18]$. In this method, the interval $[0,1]$ is divided into $N^{\text {sub }}$ subintervals at user-specified nodes, and the solution on each subinterval is approximated by a Chebyshev expansion of degree $K$, yielding $K$ th-order accuracy. If we denote the number of radial grid points by $N_{r}=K N^{s u b}$, then the total number of grid points in the disk is $M=N_{r}(2 N+1)$ and the cost of the solution to the Poisson equation is proportional to $M \log M$. 
4. Steady flows at low Reynolds number. In order to solve the steady, nonlinear Navier-Stokes equation (6), an iterative method is needed. The simplest approach is to let the nonlinear term depend on the solution at the previous iteration and solve a sequence of forced biharmonic problems until convergence is reached. In short, given the current approximation $\Psi^{k}$, we solve

$$
\begin{aligned}
\Delta^{2} \Psi^{k+1} & =-\operatorname{Re} J_{r}\left[\Psi^{k}, \Delta \Psi^{k}\right] & & \text { for } r<1, \\
\nabla \Psi^{k+1} & =\left(f^{1}(\theta), f^{2}(\theta)\right) & & \text { for } r=1,
\end{aligned}
$$

to obtain the next iterate. For the initial guess, we take $\Psi^{0}(r, \theta)$ to be the solution of the Stokes problem

$$
\begin{aligned}
\Delta^{2} \Psi^{0} & =0 & & \text { for } r<1, \\
\nabla \Psi^{0} & =\left(f^{1}(\theta), f^{2}(\theta)\right) & & \text { for } r=1,
\end{aligned}
$$

calculated by the method described in section 3 . The iteration is continued until the following convergence criterion is met:

$$
\frac{\left\|\nabla \Psi^{k+1}-\nabla \Psi^{k}\right\|}{\left\|\nabla \Psi^{k+1}\right\|}<\text { TOL } .
$$

Letting $b^{k}(r, \theta) \equiv-\operatorname{Re} J_{r}\left[\Psi^{k}, \Delta \Psi^{k}\right]$, we could choose to represent $\Psi^{k+1}$ as the sum of the initial guess $\Psi^{0}$ and the function

$$
\tilde{\Psi}^{k+1}(r, \theta)=\iint_{D} b^{k}\left(r^{\prime}, \theta^{\prime}\right) \tilde{G}_{B}\left(r, \theta ; r^{\prime}, \theta^{\prime}\right) d r^{\prime} d \theta^{\prime},
$$

where $\tilde{G}_{B}\left(r, \theta ; r^{\prime}, \theta^{\prime}\right)$ is a biharmonic Green's function satisfying $\nabla \tilde{G}_{B}\left(1, \theta ; r^{\prime}, \theta^{\prime}\right)=0$. This Green's function is available analytically in the case of a disk [9] and the above procedure is precisely that suggested by Mills [22]. Unfortunately, this approach cannot be applied to general regions. In order for our method to be extensible, we use a two-step procedure. First, we compute a "particular solution" $\Psi^{P}$ which satisfies the partial differential equation in (16), but ignores the boundary conditions. We then add a function $\Psi^{H}$ which satisfies the homogeneous Stokes equation and which is designed to correct the boundary conditions. In this representation,

$$
\Psi^{k+1}(r, \theta)=\Psi^{P}(r, \theta)+\Psi^{H}(r, \theta),
$$

we have chosen to obtain a particular solution $\Psi^{P}$ in terms of the solution to two iterated Poisson equations. We let

$$
\left\{\begin{array}{cc}
\Delta \omega^{P}=-b^{k} & \text { for } r<1 \\
\omega^{P}=0 & \text { for } r=1
\end{array}\right\}, \quad\left\{\begin{array}{cc}
\Delta \Psi^{P}=-\omega^{P} & \text { for } r<1 \\
\Psi^{P}=0 & \text { for } r=1
\end{array}\right\} .
$$

Once $\Psi^{P}$ is known, we define $\Psi^{H}$ as the solution to

$$
\begin{array}{cc}
\Delta^{2} \Psi^{H}=0 & \text { for } r<1, \\
\nabla \Psi^{H}=\left(f^{1}(\theta), f^{2}(\theta)\right)-\nabla \Psi^{P} & \text { for } r=1 .
\end{array}
$$

The problems (20) and (21) can be solved by the methods of section 3 . Thus, the work required to obtain each new fixed-point iterate $\Psi^{k+1}$ is of the order $O(M \log M)$, where $M$ is the total number of grid points in the discretization. 
5. Steady flows at higher Reynolds number. As the Reynolds number increases, the simple fixed-point iteration scheme discussed in the previous section converges more and more slowly and eventually diverges. Improvements in the convergence rate can be obtained by the introduction of relaxation factors $[6,16,22]$, but in order to handle advection-dominated flows, a more robust scheme is needed. An obvious candidate is Newton's method, which guarantees quadratic convergence for approximations sufficiently close to the desired solution. It has been used in vast numbers of studies of the Navier-Stokes equations, and we mention only a few: Fornberg [8] and Huang and Tang [14] consider flows outside and inside the unit disk, and Eisenstat and Walker [7] consider the driven cavity problem. All three of these papers use finite difference methods in either stream function-vorticity or pure stream function formulations. The computational cost of each Newton iteration is significantly greater than for the fixed-point scheme, but this increase in cost is offset by a dramatic reduction in the number of iterations required at Reynolds numbers as low as $R e=5$.

In this section, we develop a Newton iteration based on solving the fourth-order stream function equation (6) using an integral equation formulation. As in the fixedpoint case, we begin with an initial guess $\Psi^{0}(r, \theta)$ which solves the Stokes equations satisfying the boundary condition (3). Given the current guess $\Psi^{k}$, each new iterate is obtained by computing the Newton update $\delta(r, \theta)$ :

$$
\Psi^{k+1}(r, \theta)=\Psi^{k}(r, \theta)+\delta(r, \theta) .
$$

Substituting the above expression into (6) and linearizing about the current solution, it is easy to show that $\delta$ must satisfy the equation

$$
\begin{aligned}
\Delta^{2} \delta-\operatorname{Re} J_{r}\left[\Delta \delta, \Psi^{k}\right]+\operatorname{Re} J_{r}\left[\delta, \Delta \Psi^{k}\right] & =s^{k}(r, \theta) & & \text { for } r<1, \\
\nabla \delta & =(0,0) & & \text { for } r=1,
\end{aligned}
$$

where $s^{k}$ is the residual from the previous iteration

$$
s^{k} \equiv-\Delta^{2} \Psi^{k}-\operatorname{Re} J_{r}\left[\Psi^{k}, \Delta \Psi^{k}\right] .
$$

The iteration is continued until the convergence criterion (18) is met.

Equation (22) is a fourth-order, nonconstant coefficient, partial differential equation. On a uniform $N \times N$ mesh, the condition number of the linear system resulting from a finite difference discretization is proportional to $N^{4}$, resulting in significant loss of precision and posing serious convergence problems for standard iterative methods, especially in complex geometries.

We choose to seek $\delta$ in the form

$$
\delta(r, \theta)=\delta^{P}(r, \theta)+\delta^{H}(r, \theta),
$$

where

$$
\delta^{P}(r, \theta)=\iint_{D} \rho\left(r^{\prime}, \theta^{\prime}\right) G_{B}\left(r, \theta ; r^{\prime}, \theta^{\prime}\right) r^{\prime} d r^{\prime} d \theta^{\prime}
$$

$G_{B}\left(r, \theta ; r^{\prime}, \theta^{\prime}\right)$ is some biharmonic Green's function, $\rho(r, \theta)$ is an unknown volume density, and $\delta^{H}(r, \theta)$ satisfies the homogeneous biharmonic equation with boundary conditions

$$
\nabla \delta^{H}(1, \theta)=-\nabla \delta^{P}(1, \theta)
$$


While a variety of biharmonic Green's functions can be used in the representation (24), we will use the Green's function which satisfies the homogeneous boundary conditions $G_{B}\left(1, \theta ; r^{\prime}, \theta^{\prime}\right)=\Delta G_{B}\left(1, \theta ; r^{\prime}, \theta^{\prime}\right)=0$.

Substituting (23) into (22) yields the equation that determines the volume density $\rho:$

$$
\begin{array}{rlrl}
\rho+K\left(\Delta \delta^{P}, \delta^{P}\right)+K\left(\Delta \delta^{H}, \delta^{H}\right) & =s^{k} & & \text { for } r<1, \\
\nabla \delta^{P}+\nabla \delta^{H} & =(0,0) & \text { for } r=1,
\end{array}
$$

where $K(a, b)=-\operatorname{Re} J_{r}\left[a, \Psi^{k}\right]+\operatorname{Re} J_{r}\left[b, \Delta \Psi^{k}\right]$. While somewhat unwieldy if written out in full, $K\left(\Delta \delta^{P}, \delta^{P}\right)+K\left(\Delta \delta^{H}, \delta^{H}\right)$ is an expression involving integral operators with weakly singular kernels and the unknown density $\rho$. Thus, equation (25) is a Fredholm equation of the second kind. Note that $\delta^{H}$ is defined implicitly in terms of $\rho$ through the relation $\nabla \delta^{H}(1, \theta)=-\nabla \delta^{P}(1, \theta)$.

If we let $\omega^{P} \equiv \Delta \delta^{P}$, then clearly

$$
\left\{\begin{aligned}
\Delta \omega^{P}=\rho & \text { for } r<1 \\
\omega^{P}=0 & \text { for } r=1
\end{aligned}\right\}, \quad\left\{\begin{aligned}
\Delta \delta^{P} & =\omega^{P} & & \text { for } r<1 \\
\delta^{P} & =0 & & \text { for } r=1
\end{aligned}\right\} \text {. }
$$

Thus, given a volume density $\rho$, we can compute $\omega^{P}$ and then $\delta^{P}$ using our Poisson solver. Our Stokes solver can then be used to obtain $\delta^{H}$.

We discretize $\rho$ using the same polar grid as before,

$$
\left(r_{i}, \theta_{j}\right), \quad 1 \leq i \leq N_{r}, \quad 1 \leq j \leq 2 N+1,
$$

associating an unknown density value $\rho_{i j}$ with each location. Equation (25) then becomes a dense, finite-dimensional linear system for these values. By contrast with a finite difference or finite element formulation, this linear system has a bounded condition number as $N_{r}, N \rightarrow \infty$. The representation (23) can, in fact, be interpreted as preconditioning the partial differential equation (22) with the biharmonic equation. (Eisenstat and Walker [7] use such a preconditioner explicitly in the finite difference context.) As a result, the discrete version of (25) can be solved in a straightforward manner using the generalized minimum residual method (GMRES) [27]. The bulk of the work at each GMRES iteration is the "matrix-vector product." From the preceding discussion, it is clear that, given the vector of density values $\vec{\rho}=\left\{\rho_{i j}\right\}$, each iteration requires the solution of two Poisson equations (26) to obtain $\delta^{P}[\vec{\rho}]$ and $\omega^{P}[\vec{\rho}]$ and the solution of one biharmonic equation to compute $\delta^{H}$. Thus, each iteration for the linearized problem (25) requires more or less the same amount of work as a single fixed-point iteration for the nonlinear problem (16).

An iterative technique, such as GMRES, is equipped with a parameter tol which specifies the precision to which equation (25) is solved. In a classical Newton method, tol would normally remain fixed throughout the (outer) Newton iteration at some value smaller than the (outer) Newton tolerance TOL. However, it is pointless to solve (25) to high precision when the iterate $\Psi^{k}$ is far from the exact solution. Inexact Newton methods are obtained by allowing tol to vary with each outer iteration, becoming more and more stringent as the iterates converge to the desired solution [7]. It can be shown that quadratic convergence is retained. An obvious choice is to define the tolerance for the $k$ th iterate by

$$
t_{o l}^{k}=\max \left(\epsilon_{1} T O L, \epsilon_{2}\left\|s^{k}(r, \theta)\right\|\right),
$$


where $\epsilon_{1}, \epsilon_{2}$ are small constants. In our experience, the condition (27) with $\epsilon_{1}=10^{-2}$ and $\epsilon_{2}=10^{-1}$ reduces the total amount of work by a factor of 2 when compared with the strategy $t o l^{k}=\epsilon_{1} \cdot T O L$. The gains in performance, of course, depend on the detailed behavior of the Newton iteration. The more steps required before rapid convergence sets in, the greater the speedup obtained from strategies such as (27).

6. Implicit methods for unsteady flows. We turn now to the solution of the unsteady Navier-Stokes equations expressed in the form (5) which contains both diffusive and convective terms. At moderate values of Re, "linearly implicit" or IMEX schemes are generally used for marching in time. In such schemes, the diffusive term $(1 / R e) \Delta^{2} \Psi$ is treated implicitly, while the convective term $J_{r}[\Psi, \Delta \Psi]$ is treated explicitly. At each time step, one must solve a linear elliptic equation, but one avoids the severe diffusive stability requirements imposed on a fully explicit scheme. The remaining stability constraint is dictated by a CFL condition, which in turn depends on the velocity field. At sufficiently high Reynolds numbers, this CFL condition can be dominant, but we are interested in problems where diffusion plays an important role and for which explicit methods are ineffective.

Ascher, Ruuth, and Wetton [2] present a systematic approach, based on stability arguments, for selecting and developing suitable IMEX schemes for a range of Reynolds numbers and a variety of spatial discretizations. Regardless of the details of the particular choice of IMEX scheme, time discretization of (5) yields the linear partial differential equation:

$$
\begin{aligned}
\Delta \Psi^{N+1}-\alpha \Delta^{2} \Psi^{N+1} & =b\left(\Psi^{N}, \Psi^{N-1}, \ldots\right) & & \text { for } r<1, \\
\nabla \Psi^{N+1} & =\left(f^{1}(t, \theta), f^{2}(t, \theta)\right) & & \text { for } r=1, \\
\Psi^{0} & =\Psi_{0}(r, \theta) & & \text { at } t=0,
\end{aligned}
$$

where $t=(N+1) \Delta t$ is the current time. The simplest such scheme is the first-order backward Euler method, which is of the form (28) with

$$
\alpha=\frac{\Delta t}{R e}, \quad b=\Delta \Psi^{N}+\Delta t J_{r}\left[\Psi^{N}, \Delta \Psi^{N}\right] .
$$

A second-order method shown in [2] to be suitable for low to moderate values of the Reynolds number is the extrapolated Gear method (EXG), for which

$$
\alpha=\frac{2 \Delta t}{3 R e}, \quad b=\frac{4}{3} \Delta \Psi^{N}-\frac{1}{3} \Delta \Psi^{N-1}+\frac{4}{3} \Delta t J_{r}^{N}-\frac{2}{3} \Delta t J_{r}^{N-1},
$$

where $J_{r}^{N}=J_{r}\left[\Psi^{N}, \Delta \Psi^{N}\right]$. At higher Reynolds number, a less diffusive scheme is often used, such as Crank-Nicolson/leap frog (CNLF), for which

$$
\alpha=\frac{\Delta t}{R e}, \quad b=\Delta \Psi^{N-1}+2 \Delta t J_{r}^{N}+\frac{\Delta t}{R e} \Delta^{2} \Psi^{N-1} .
$$

In order to solve the modified biharmonic equation (28) by means of integral equations, we first represent the solution at each time step as

$$
\Psi^{N+1}(r, \theta)=\Psi^{P}(r, \theta)+\Psi^{H}(r, \theta),
$$

where

$$
\left\{\begin{aligned}
\omega^{P}-\alpha \Delta \omega^{P}=-b & \text { for } r<1 \\
\omega^{P}=0 & \text { for } r=1
\end{aligned}\right\}, \quad\left\{\begin{array}{cc}
\Delta \Psi^{P}=-\omega^{P} & \text { for } r<1 \\
\Psi^{P}=0 & \text { for } r=1
\end{array}\right\}
$$


and $\Psi^{H}$ satisfies

$$
\begin{array}{cc}
\Delta \Psi^{H}-\alpha \Delta^{2} \Psi^{H}=0 & \text { for } r<1, \\
\nabla \Psi^{H}=\left(f^{1}(t, \theta), f^{2}(t, \theta)\right)-\nabla \Psi^{P} & \text { for } r=1 .
\end{array}
$$

The Poisson solver discussed in section 3 is easily adapted to solve the equations (30) to determine $\omega^{P}$. Slightly more complicated is the equation (31), for which the general solution in a disk can be expressed as

$$
\Psi^{H}=\sum_{n=-\infty}^{\infty}\left[A_{n} I_{n}\left(\frac{r}{\sqrt{\alpha}}\right)+B_{n} r^{|n|}\right] e^{i n \theta},
$$

where $I_{n}$ are the modified Bessel functions of the first kind. The coefficients $A_{n}$ and $B_{n}$ are found from the boundary conditions. To carry this out, let $\left(g^{1}(\theta), g^{2}(\theta)\right)=$ $\left(f^{1}(t, \theta), f^{2}(t, \theta)\right)-\nabla \Psi^{P}(t, 1, \theta)$, which is represented in complex notation as $g(\theta)=$ $g^{1}(\theta)+i g^{2}(\theta)$. We approximate this by the truncated Fourier series

$$
g(\theta) \approx \sum_{n=-N}^{N} g_{n} e^{i n \theta} .
$$

Matching coefficients of $e^{i n \theta}$ yields

$$
\begin{array}{ccc}
A_{0}=\frac{\sqrt{\alpha} g_{0}}{I_{1}(1 / \sqrt{\alpha})}, \quad A_{n}=\frac{\sqrt{\alpha} g_{n}}{I_{n+1}(1 / \sqrt{\alpha})}, \quad A_{n}=\overline{A_{-n}}, & 1<n \leq N, \\
B_{n}=\frac{1}{2 n}\left[\frac{A_{n}}{\sqrt{\alpha}} I_{n+1}\left(\frac{1}{\sqrt{\alpha}}\right)-g_{n}\right], \quad B_{n}=\overline{B_{-n}}, & -N<n \leq-1 .
\end{array}
$$

Clearly, the amount of work required at each time step is of the order $O(M \log M)$ for a polar grid with $M$ discretization points.

7. Numerical results. The algorithms described above have been implemented in Fortran. Here, we illustrate their performance on a variety of interior-flow examples. All timings cited are for a Sun SPARC-20 workstation.

Example 1: An inflow-outflow problem. We first consider a setting in which fluid is injected radially over one portion of the disk boundary and ejected radially over another. This type of flow is occasionally used as a model for the ventilation of confined spaces (see [22] and the references therein), and the boundary conditions are typically of the form

$$
u_{r}(1, \theta)=\left\{\begin{array}{ll}
1 / \epsilon^{\prime}, & \alpha-\epsilon^{\prime}<\theta<\alpha+\epsilon^{\prime}, \\
0, & \alpha+\epsilon^{\prime}<\theta<\beta-\epsilon, \\
-1 / \epsilon, & \beta-\epsilon<\theta<\beta+\epsilon, \\
0, & \beta+\epsilon<\theta<2 \pi+\alpha-\epsilon^{\prime},
\end{array} \quad u_{\theta}(1, \theta)=0 .\right.
$$

While it is clear that this velocity distribution satisfies the zero net flux condition (4), the boundary condition is discontinuous. In order to simplify the problem and retain high-order convergence from the Fourier discretization of $u_{r}(1, \theta)$, we mollify the velocity distribution (32) and use

$$
u_{r}(1, \theta)=\frac{4}{\sqrt{\pi} \epsilon} \begin{cases}e^{-4(\theta-\alpha)^{2} / \epsilon^{2}}, & \alpha-6 \epsilon<\theta<\alpha+6 \epsilon \\ 0, & \alpha+6 \epsilon<\theta<\beta-6 \epsilon \\ -e^{-4(\theta-\beta)^{2} / \epsilon^{2}}, & \beta-6 \epsilon<\theta<\beta+6 \epsilon \\ 0, & \beta+6 \epsilon<\theta<2 \pi+\alpha-6 \epsilon .\end{cases}
$$




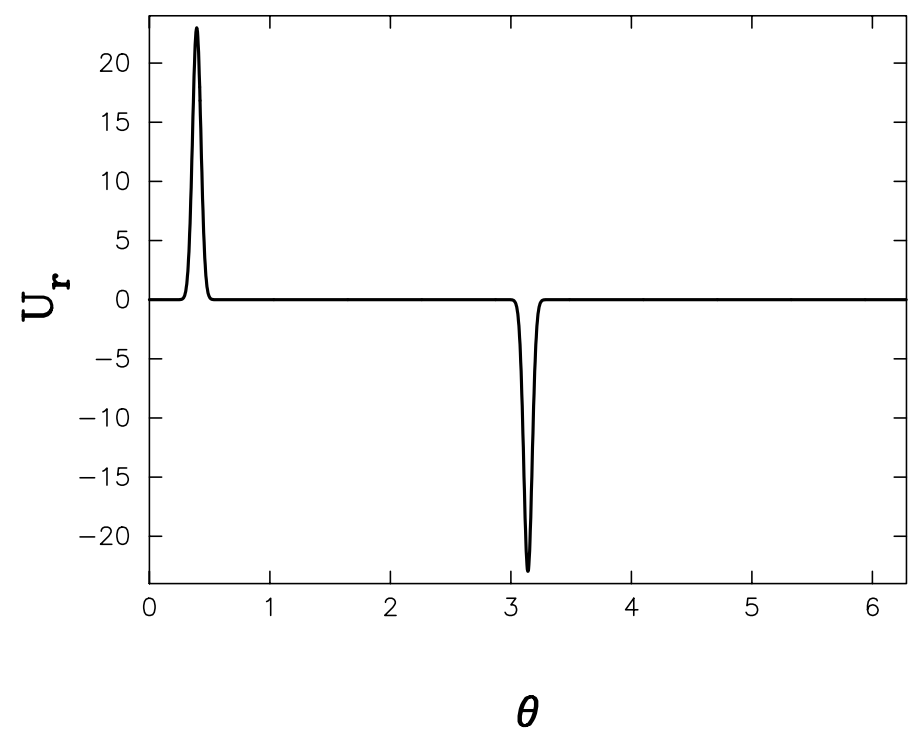

FIG. 1. Velocity boundary conditions for the inflow-outflow problem of Example 1.
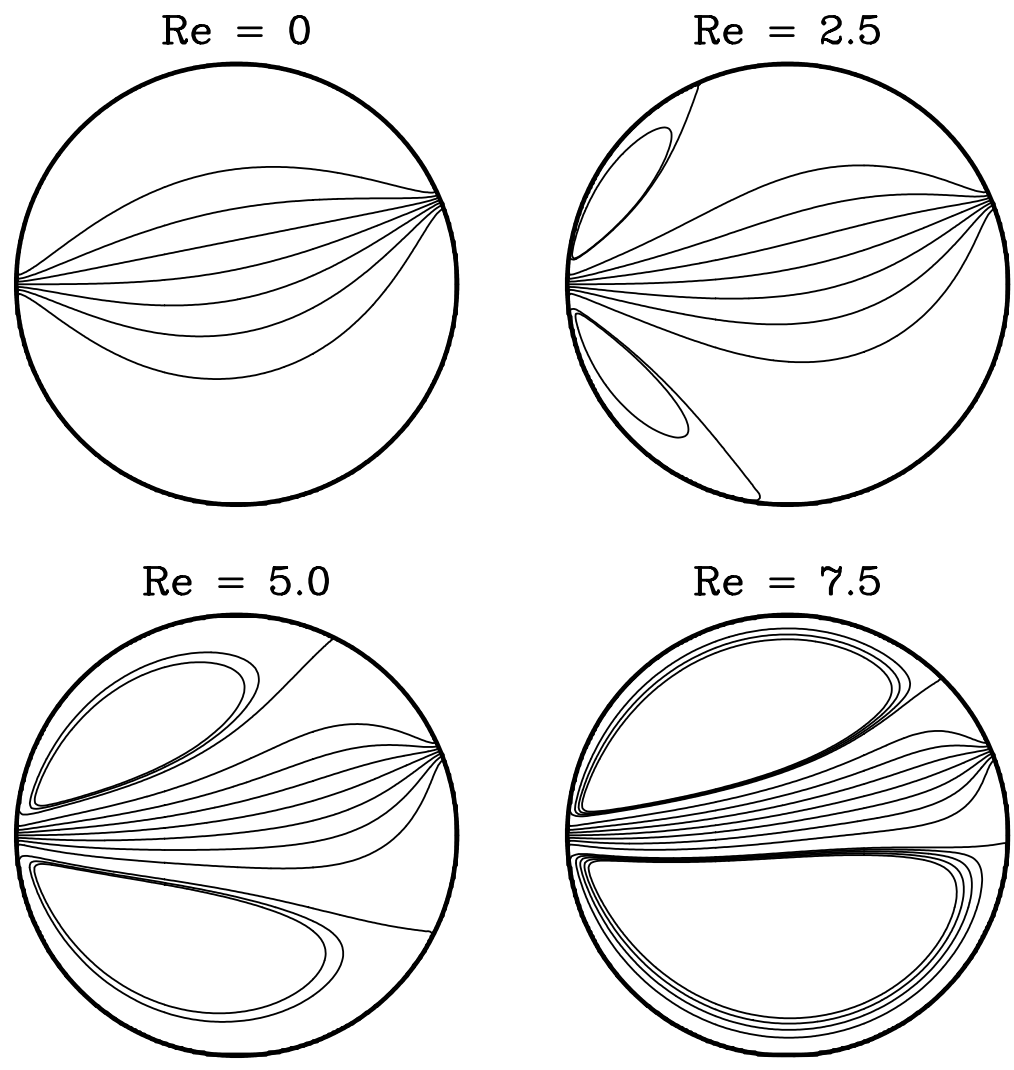

FIG. 2. Streamlines of the solution to the inflow-outflow problem of Example 1 at various Reynolds numbers. The inflow region is centered at $\theta=\pi$, the outflow region is centered at $\theta=\pi / 8$, and the length of both segments is $\pi / 16$. The computational grid was uniform with $N^{s u b}=40$, $K=4$, and $N=150$. 
TABLE 1

Comparison of the fixed-point and inexact Newton methods for solving the inflow-outflow problem of Example 1. In these calculations, $N^{s u b}=40, K=4$, and $N=150$. The fixed-point iteration diverges for $R e>3$.

\begin{tabular}{|r||r|r||r|r||}
\hline \multicolumn{1}{|c||}{ Re } & \multicolumn{2}{c||}{ Fixed point } & \multicolumn{2}{|c||}{ Inexact newton } \\
\hline 0.5 & 5 & 73 & 4 & \# Iterations \\
1.0 & 6 & 87 & 5 & 219 \\
1.5 & 7 & 102 & 15 & 392 \\
2.0 & 9 & 131 & 15 & 392 \\
2.5 & 11 & 160 & 15 & 392 \\
3.0 & 19 & 258 & 29 & 625 \\
3.5 & - & - & 31 & 654 \\
4.0 & - & - & 49 & 947 \\
5.0 & - & - & 53 & 1006 \\
7.5 & - & - & 128 & 2367 \\
\hline
\end{tabular}

In Figure 1, the velocity profile is shown for $\epsilon=\epsilon^{\prime}=\pi / 32, \alpha=\pi / 8$, and $\beta=\pi$.

Figure 2 shows the solution at several values of the Reynolds number. Our results appear to be in good agreement with those calculated in [6, 14, 22]. Of particular note are the calculations of Huang and Tang [14], who were able to carry out this computation for Reynolds numbers up to 1000 using an adaptive method developed specifically for this application. We have limited our calculations to a grid with 160 points in the radial direction and 301 points in the azimuthal direction, which is sufficient to resolve flows with Reynolds numbers up to 7.5.

Table 1 compares the number of iterations and total CPU time required for both the fixed-point scheme and the inexact Newton method, using a tolerance $T O L=$ $10^{-6}$. To accelerate convergence, we use a continuation method with respect to Re. In other words, as we increase the Reynolds number, the solution obtained with the preceding value of $R e$ is used as the initial guess, rather than the Stokes flow solution $(R e=0)$. The number of iterations indicated in the table for Newton's method refers to the total number of inner GMRES iterations. No more than 10 outer Newton iterations are needed for each stage.

Example 2: Batchelor's constant-vorticity theorem. An interesting class of problems, called moving-wall problems, consist of steady flows inside a disk generated by tangential motion of part or all of the boundary:

$$
u_{r}(1, \theta)=0, \quad u_{\theta}(1, \theta)=-f^{1}(\theta) .
$$

If $f^{1}(\theta)$ is constant, the steady solution is that of rigid-body rotation. As a simple model for recirculating flows in physical cavities at high Reynolds number, Batchelor [3] has suggested letting $f^{1}(\theta)$ be constant over a portion of the arc and zero elsewhere. In order to test our approach in a slightly simpler setting, we let $f^{1}(\theta)$ vary smoothly. In the present example, we consider the internal flow generated by

$$
u_{r}(1, \theta)=0, \quad u_{\theta}(1, \theta)=-\frac{1}{2}(1+\cos \theta),
$$

a problem solved by Kuwahara and Imai [16], for $R e \leq 1024$, and by Mills [22], for $R e \leq 64$. 

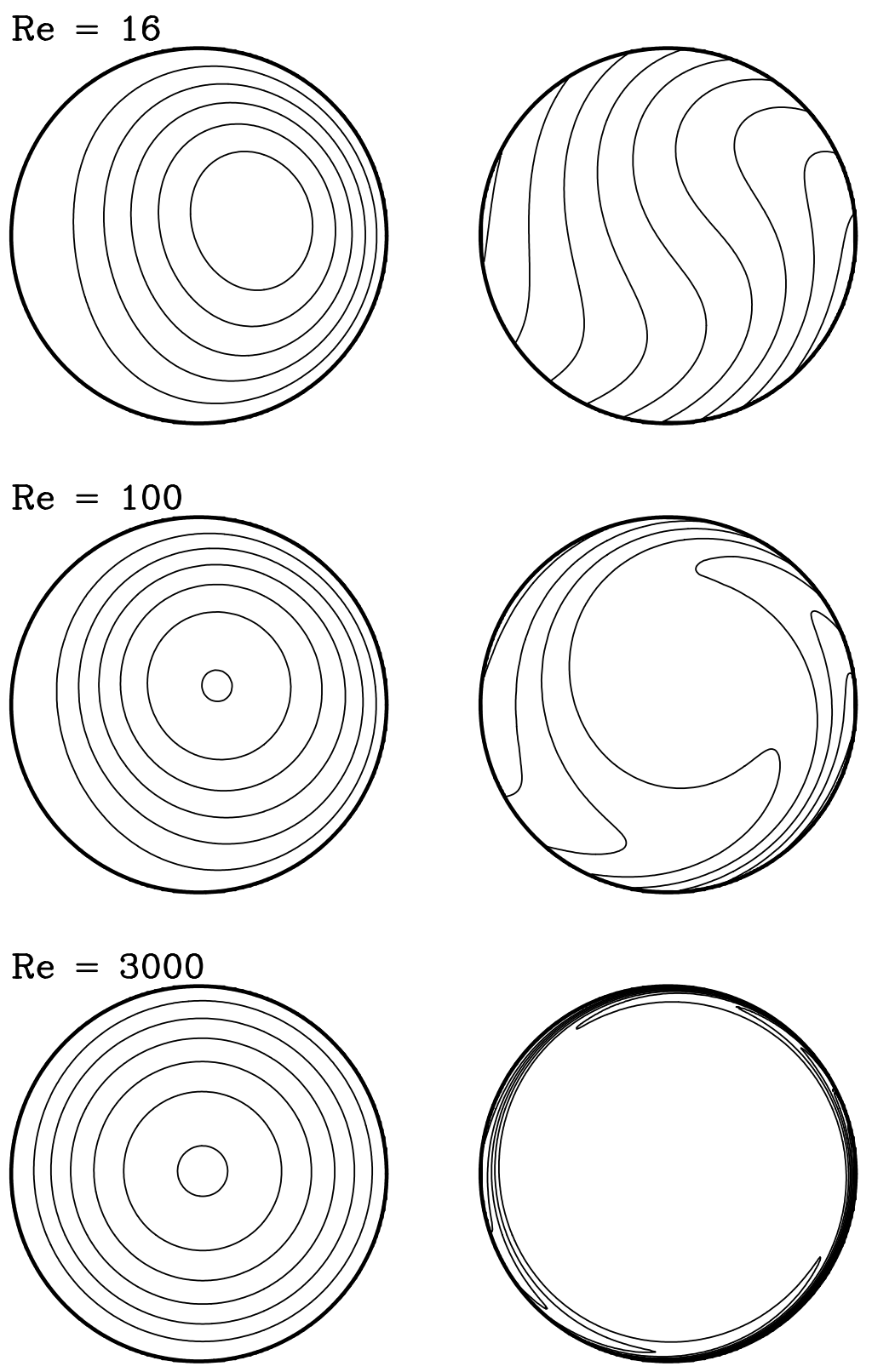

FIG. 3. Solutions to the moving-wall problem of Example 2 at several values of the Reynolds number. The left-hand plots show streamlines of the solution, while the right-hand plots show contours of vorticity. In these calculations, $N^{s u b}=30, K=8$, and $N=16$.

This example serves as a nice illustration of the behavior of steady closed flows as $R e \rightarrow \infty$. In this limit, the region in which viscous forces dominate shrinks to a layer on the boundary and, as described by Batchelor's constant-vorticity theorem, the vorticity is uniform in a connected region of small viscous forces. The value of this "core" vorticity can be computed from a boundary layer solution $[4,15,31]$. Without entering into details, the idea is that the region with constant vorticity is behaving like 


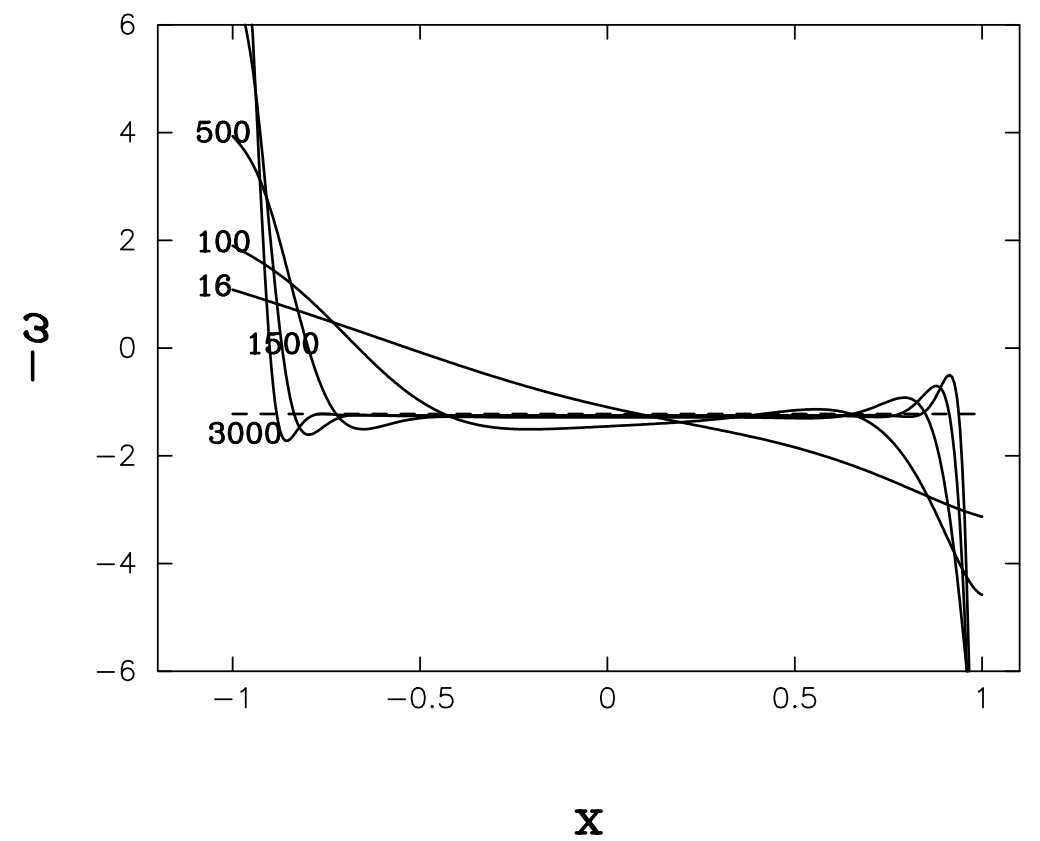

FIG. 4. Vorticity distribution along the $x$ axis at several values of the Reynolds number for the moving-wall problem of Example 2. The horizontal dashed line represents the value of the vorticity at the core as predicted by Batchelor's theorem.

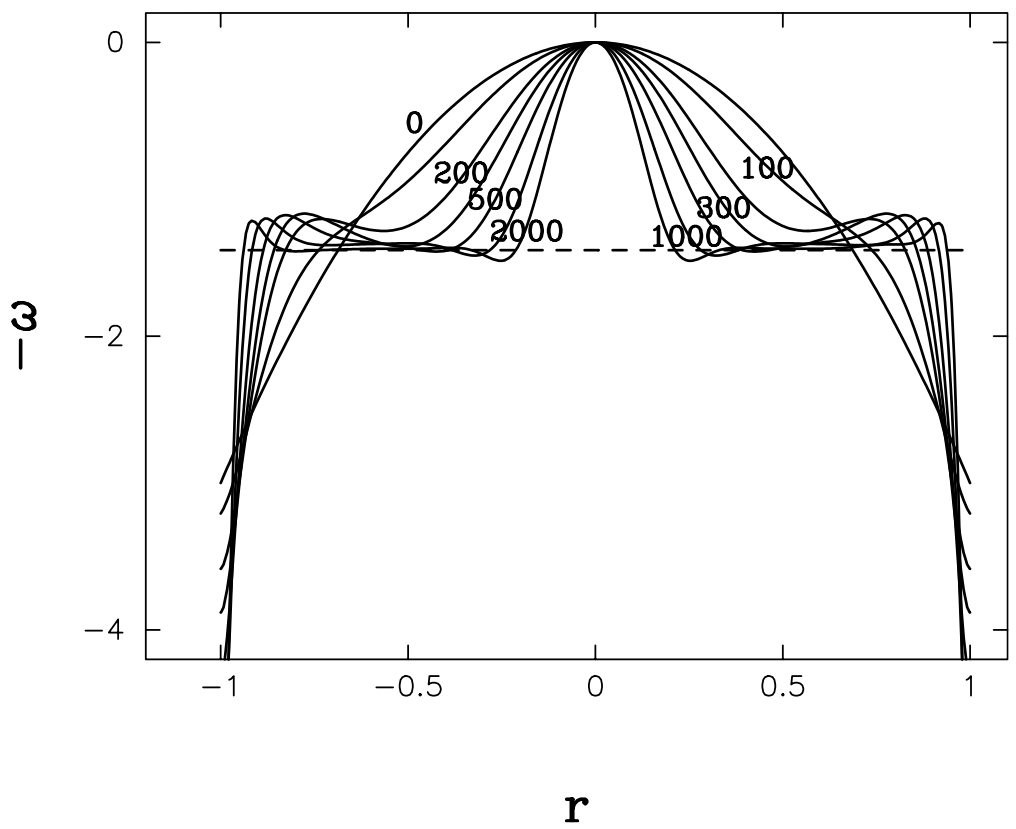

FIG. 5. Vorticity distribution along the diameter $x=y$ of the unit disk at several values of the Reynolds number for the moving-wall problem of Example 3. The horizontal line represents the value of the vorticity at the two cores as predicted by Batchelor's theorem. 

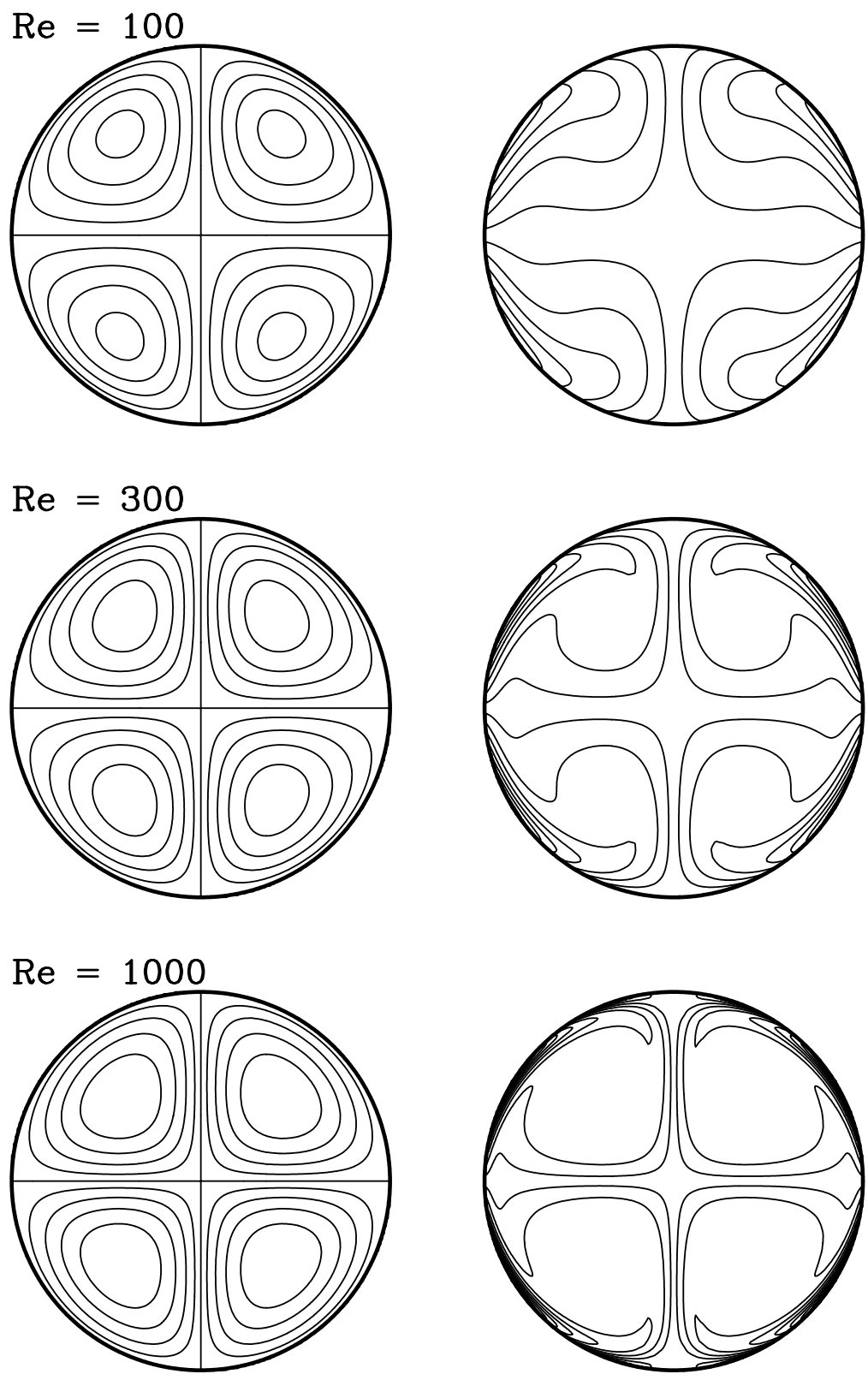

Fig. 6. Solutions to the moving-wall problem of Example 3 at several values of the Reynolds number. The left-hand plots show streamlines of the solution and the right-hand plots show contours of vorticity. In these calculations, $N^{s u b}=40, K=4$, and $N=64$.

a rigid-body rotation, $u_{\theta}=\omega_{0} / 2$, where $\omega_{0}$ is the core vorticity. Thus, one can equate integrals of the velocity along the physical boundary with integrals of the velocity just inside the boundary layer:

$$
\int_{0}^{2 \pi} u_{\theta}^{2}(1, \theta) d \theta=\int_{0}^{2 \pi} u_{\theta}^{2}(1-\delta, \theta) d \theta
$$


TABLE 2

Performance of the inexact Newton method for the moving-boundary problem of Example 3. In the first column, we list the Reynolds number. In the second column, we list the number of Newton iterations required to solve the steady-state Navier-Stokes equations, using the solution at the preceding Reynolds number as the initial guess. In the third column, we list the total number of GMRES iterations needed at each Reynolds number, and in the fourth column, we list the amount of CPU time expended.

\begin{tabular}{|r|c|r|r|}
\hline$R e$ & $\begin{array}{c}\text { Newton } \\
\text { iterations }\end{array}$ & $\begin{array}{r}\text { GMRES } \\
\text { iterations }\end{array}$ & Time \\
\hline 100 & 4 & 42 & 310.0 \\
200 & 4 & 85 & 626.6 \\
300 & 4 & 131 & 974.1 \\
400 & 4 & 180 & 1349.4 \\
500 & 4 & 231 & 1737.8 \\
600 & 4 & 270 & 2037.7 \\
700 & 4 & 307 & 2325.9 \\
800 & 3 & 191 & 1417.0 \\
900 & 4 & 414 & 3248.8 \\
1000 & 4 & 440 & 3421.6 \\
\hline
\end{tabular}

where $\delta$ is the width of the boundary layer. The first integral is known from the boundary condition $u_{\theta}(1, \theta)=-\frac{1}{2}(1+\cos \theta)$, and we use the approximation $u_{\theta}(1-$ $\delta, \theta) \approx \omega_{0} / 2$ to determine the unknown value of the core vorticity. A straightforward calculation yields the asymptotic result

$$
\lim _{R e \rightarrow \infty}\left|\omega_{0}\right|=\sqrt{3 / 2} .
$$

Figure 3 demonstrates this convergence to a core of constant vorticity quite clearly. Figure 4 plots the vorticity distribution along the $x$ axis of the cylinder for several values of $R e$, and shows that the vorticity away from the boundary layer is converging to a uniform distribution at the predicted value.

The steady equations were solved using the fixed-point scheme until $R e \approx 20$. For higher Reynolds numbers, we used the inexact Newton method. Detailed timing results are presented for the next, somewhat more complicated, example.

Example 3: A moving-wall problem with multiple cell formation. In the preceding example, the boundary motion results in a single cell of constant vorticity as $R e \rightarrow \infty$. By changing the boundary conditions, it is possible to get more than one cell within the cylinder, each having a different core vorticity as $R e \rightarrow \infty$. Here, we consider the motion generated by

$$
u_{r}(1, \theta)=0, \quad u_{\theta}(1, \theta)=-\cos \theta \sin \theta .
$$

The azimuthal velocity changes sign four times on the boundary, and as can be seen in Figure 6, the cylinder breaks up into four quadrants each enclosed by the streamline $\Psi=0$. Figure 5 shows the vorticity distribution along the diameter of the cylinder $x=y$ at increasing values of $R e$. The vorticity does tend toward a core value which is extracted numerically to be approximately 1.4. Detailed timing information is given in Table 2 .

Note that the number of Newton iterations remains more or less constant as the Reynolds number increases, but that the total number of GMRES iterations required grows linearly. This is a reflection of the fact that the partial differential equation (22) which is solved at each Newton step becomes more and more advection dominated, while our current preconditioner is based on Stokes flow. Improved iterative strategies 

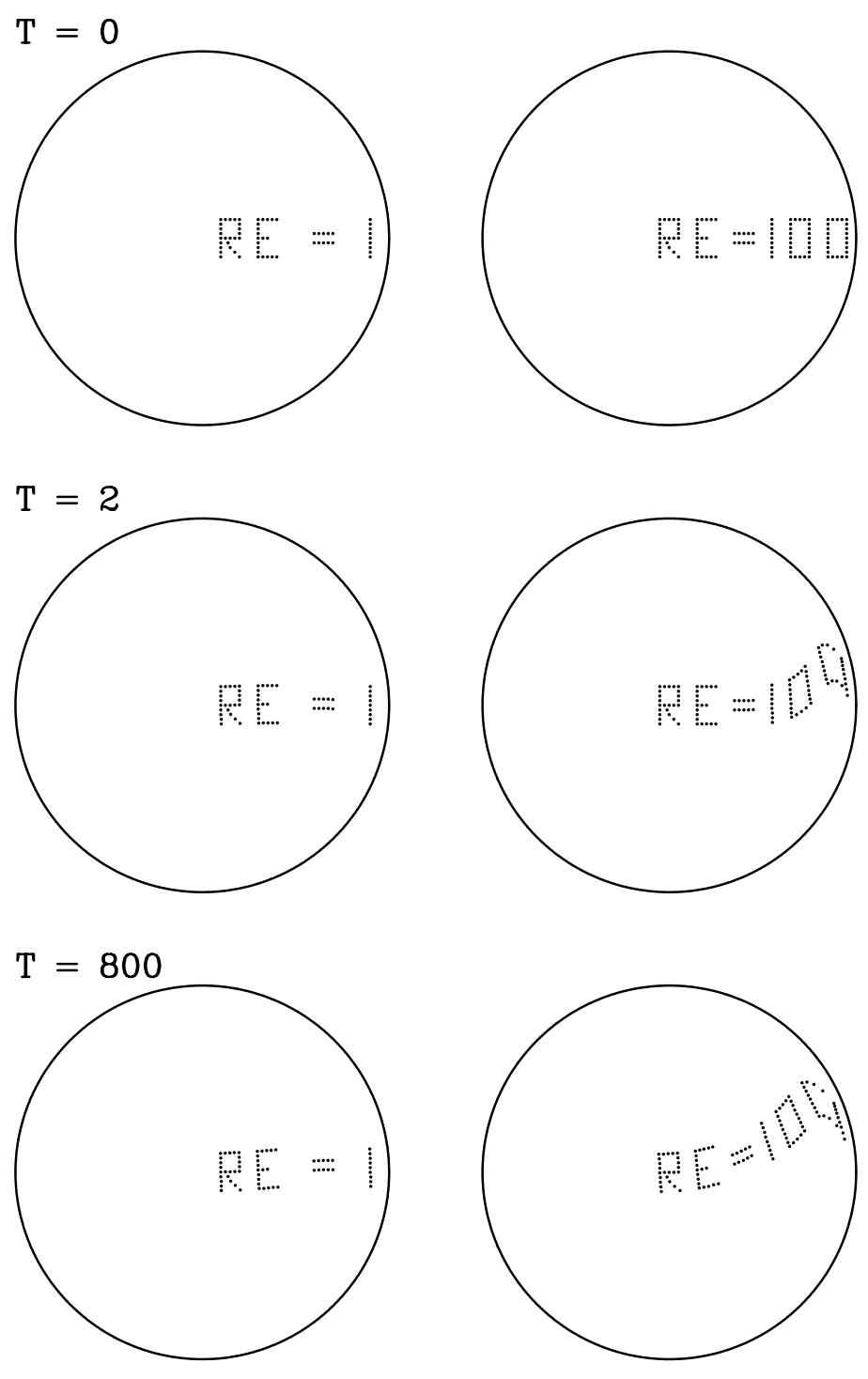

FIG. 7. Solutions to the washing machine problem of Example 4 at two different Re. The upper plots show the particle distribution at $t=0$; the middle plots show their positions after one cycle $(t=2)$; and the bottom plots are the positions after 400 cycles. In these calculations, $N^{s u b}=20$, $K=8, N=16$, and $\Delta t=0.1$.

will have to be incorporated if higher Reynolds numbers are to be reached in a practical setting.

Example 4: A washing machine. For the final example, we consider an unsteady flow in which a fluid, initially at rest, is subject to a periodic rotation of the boundary:

$$
u_{r}(1, \theta, t)=0, \quad u_{\theta}(1, \theta, t)=-\operatorname{signum}[\sin (\pi t)] .
$$

We use the extrapolated Gear method (29) as an evolution scheme and visualize the flow by tracking "particles" which move passively with the fluid according to 


$$
\frac{d \mathbf{x}}{d t}=\mathbf{u}
$$

The results of this simulation are shown in Figure 7. Note that at $R e=1$, the particles return more or less to their initial configuration even after 400 cycles. This is a consequence of the nearly negligible inertial effects. For $R e=100$, the particles near the boundary do not return to their starting positions even after one cycle, and this distortion moves slowly inward as the number of cycles progresses.

8. Discussion. We have presented a class of integral equation methods for the solution of the steady and unsteady incompressible Navier-Stokes equations in two space dimensions. These methods eliminate the need to enforce the divergence-free condition on the velocity field at each step, as in projection methods, or generating boundary conditions for the vorticity, as in stream function/vorticity methods. Although our methods rely, in a fundamental way, on fast solvers for the steady and unsteady Stokes equations, our numerical results appear quite promising for Reynolds numbers as large as $R e=3000$.

In the present paper, the Navier-Stokes equations are solved by fast direct methods specifically designed for a disk, but we believe that the integral equation viewpoint will be of benefit in a more general setting. Fast, iterative methods based on potential theory are already available for the Poisson equation and the homogeneous Stokes equations in arbitrary multiply connected domains $[10,19,20]$. With these tools, it is a straightforward matter to extend our steady-state algorithms to complex geometries [11]. For the unsteady problem, we also need a method to solve the homogeneous modified biharmonic equation (31) in general regions. As $R e \rightarrow \infty$ and $\Delta t \rightarrow 0$, this is a singularly perturbed fourth-order partial differential equation. There is a suitable potential theory for this problem [13], but we are not aware of any existing fast algorithms to make this potential theory practical. This is a topic of current research.

Acknowledgments. We would like to thank Julian Cole, Tao Tang, Weizhang Huang, and Brian Wetton for several useful discussions.

\section{REFERENCES}

[1] C. R. Anderson (1989), Vorticity boundary conditions and boundary vorticity generation for two-dimensional viscous incompressible flows, J. Comput. Phys., 80, pp. 72-97.

[2] U. M. Ascher, S. J. Ruuth, and B. T. R. Wetton (1995), Implicit-explicit methods for time-dependent partial differential equations, SIAM J. Numer. Anal., 32, pp. 797-823.

[3] G. K. BAtChELOR (1956), A proposal concerning laminar wakes behind bluff bodies at large Reynolds number, J. Fluid Mech., 1, pp. 388-398.

[4] G. K. Batchelor (1956), On steady laminar flow with closed streamlines at large Reynolds number, J. Fluid Mech., 1, pp. 177-190.

[5] R. H. Chan, T. K. DeLillo, and M. A. Horn (1997), The numerical solution of the biharmonic equation by conformal mapping, SIAM J. Sci. Comput., 18 (1997), pp. 1571-1582.

[6] S. C. R. Dennis, M. NG, And P. NGuYen (1992), Numerical solution for the steady motion of a viscous fluid inside a circular boundary using integral conditions, J. Comput. Phys., 108, pp. 142-152.

[7] S. C. Eisenstat and H. F. Walker (1996), Choosing the forcing terms in an inexact Newton method, SIAM J. Sci. Comput., 17, pp. 16-32.

[8] B. Fornberg (1985), Steady viscous flow past a circular cylinder up to Reynolds number 600 J. Comput. Phys., 61, pp. 297-320.

[9] P. R. Garabedian (1964), Partial Differential Equations, Wiley, New York.

[10] L. Greengard, M. C. Kropinski, And A. Mayo (1996), Integral equation methods for Stokes flow and isotropic elasticity in the plane, J. Comput. Phys., 125, pp. 403-414.

[11] L. Greengard, M. C. Kropinski, and A. Mayo (1997), A fast solver for the steady NavierStokes equations in two dimensions, in preparation. 
[12] L. Greengard and V. Rokhlin (1991), On the numerical solution of two-point boundary value problems, Comm. Pure Appl. Math., 44, pp. 419-452.

[13] G. Hsiao And R. C. MacCamy (1973), Solution of boundary value problems by integral equations of the first kind, SIAM Review, 15, pp. 687-705.

[14] W. Huang And T. TANG (1995), Steady motion of a viscous fluid inside a circular boundary, Internat. J. Numer. Methods Fluids, submitted.

[15] S.-C. Кім (1997), On Prandtl-Batchelor theory of a cylindrical eddy, SIAM J. Appl. Math., to appear.

[16] K. Kuwahara and I. Imai (1969), Steady, viscous flow within a circular boundary, Phys. Fluids 12 Suppl. II, pp. 94-101.

[17] W. E. Langlois (1964), Slow Viscous Flow, Macmillan, New York.

[18] J.-Y. Lee and L. Greengard (1995), A fast adaptive numerical method for stiff two-point boundary value problems, SIAM J. Sci. Comput., 18 (1997), pp. 403-429.

[19] A. MAYO (1992), The rapid evaluation of volume integrals of potential theory on general regions, J. Comput. Phys., 100, pp. 236-245.

[20] A. McKenney, L. Greengard, and A. Mayo (1995), A fast Poisson solver for complex geometries, J. Comput. Phys., 118, pp. 348-355.

[21] S. G. Mikhlin (1957), Integral Equations, Pergamon Press, London.

[22] R. D. Mills (1977), Computing internal viscous flow problems for the circle by integral methods, J. Fluid Mech., 79, pp. 609-624.

[23] S. G. Muskhelishvili (1953), Some Basic Problems of the Mathematical Theory of Elasticity, P. Noordhoff, Groningen, the Netherlands.

[24] J. M. Occhialini, G. P. Muldowney, And J. J. L. Higdon (1992), Boundary integral/spectral element approaches to the Navier-Stokes equations, Internat. J. Numer. Methods Fluids, 15, pp. 1316-1381.

[25] P. K. G. Panikker and Z. Lavan (1975), Flow past impulsively started bodies using Green's functions, J. Comp. Phys., 18, pp. 46-65.

[26] V. Z. Parton and P. I. Perlin (1982), Integral Equation Methods in Elasticity, MIR, Moscow.

[27] Y. SAAD AND M. H. SChUlTZ (1986), GMRES: a generalized minimum residual algorithm for solving nonsymmetric linear systems, SIAM J. Sci. Stat. Comput., 7, pp. 856-869.

[28] S. Timoshenko and J. N. Goodier (1951), Theory of Elasticity, McGraw-Hill, New York.

[29] J. S. Uhlman (1992), An Integral Equation Formulation of the Equations of Motion of an Incompressible Fluid, Naval Undersea Warfare Center-NPT Technical Report 10,086, Newport, RI.

[30] C. M. WANG AND J. C. Wu (1986), Numerical solution of steady Navier-Stokes problems using integral representations, AIAA J., 24, pp. 1305-1312.

[31] W. W. Wood (1957), Boundary layers whose streamlines are closed, J. Fluid Mech., 2, pp. 7787. 